KARL JANSEN-WINKELN

\title{
Lebenslehre und Biographie ${ }^{1}$
}

1. Wenn man hört, im alten Ägypten habe es einerseits „Lebenslehren“ gegeben, in denen das rechte Verhalten für Beruf und Alltagsleben gelehrt wurde, andrerseits „(Auto)Biographien“, in denen man zurückblickend über die eigene vorbildliche Lebensführung sprach, sollte man a priori einen Zusammenhang zwischen diesen beiden Arten von Texten vermuten: Etwa in dem Sinne, daß die Lebenslehren allgemeine Verhaltensregeln vermitteln, die Biographien dann deren konkrete Ausführung, die Lehren eher das Grundsätzliche beschreiben, die Biographien den Einzelfall und die Details.

Tatsächlich wird in der Literatur auch oft von einem engen Zusammenhang von Lebenslehre und Biographie gesprochen: So nennt Brunner in der Einführung zu seiner Anthologie der Weisheitslehren unter den mit den Lehren verwandten Textgattungen an erster Stelle die Biographie, die sich in ihren Ausführungen über berufliche Erfolge, besondere Erlebnisse und Charakter eng mit den Lebenslehren berühre und teilweise dieselbe Terminologie verwende: „Was in den Lehren als Empfehlung, als Rat und Forderung ausgesprochen ist, erscheint in den Biographien als Erfüllung. " Ebenso sieht es

1 Abkürzungen nach Lexikon der Ägyptologie, Bd. 7, XIII-XXXVIII. Außerdem: Brunner, Weisheit = H. Brunner, Altägyptische Weisheit, Darmstadt 1988; Gnirs, Autobiographie = A. Gnirs, „Die ägyptische Autobiographie" in: A. Loprieno (ed.), Ancient Egyptian Literature, PÄ 10, 1996, 191-241; JansenWinkeln, Ägypt. Biogr. = K. Jansen-Winkeln, Ägyptische Biographien der 22. und 23. Dynastie, ÄUAT 8, 1985; Quack, Ani = J. F. Quack, Die Lehren des Ani, OBO 141, 1994; Studien zu Lebenslehren = E. Hornung/O. Keel, Studien zu altägyptischen Lebenslehren, OBO 28, 1979; Vernus, Sagesses = P. Vernus, Sagesses de l'Egypte pharaonique, Paris 2001.

Brunner, Weisheit, 82.
P. Vernus: „On discerne immédiatement le lien entre l'autobiographie égyptienne et la sagesse. Toutes deux convoquent un ensemble de normes éthiques; la première, pour les illustrer à travers un autoportrait; la seconde, pour les légitimer à travers un enseignement. Celle-ci les pose; celle-là les présuppose ". Auch die thematischen Schwerpunkte und Entwicklungen seien in beiden Textarten ähnlich oder gleich, z. B. Loyalismus im Mittleren Reich, Frömmigkeit im späteren Neuen Reich, ebenso bestimmte prominente Einzelthemen wie etwa Elternliebe, Wohltätigkeit, Beliebtheit bei den Mitbürgern: die Lehren fordern dazu auf, die Biographien berichten es als im Einzelfall erfüllt. Auch sonst ist oft auf den engen Zusammenhang von Lebenslehre und Biographie verwiesen worden ${ }^{4}$. Man hat sogar die Biographie als Ursprung der ägyptischen Weisheitsliteratur und damit auch der Lebenslehre vermutet ${ }^{5}$. Als besonders schlagender Beweis für die enge Verwandtschaft von Biographien und Lebenslehren wird dabei öfters darauf verwiesen, daß einerseits einige der Lehren (die Lehre Amenemhets und die Lehre für Merikare) in Teilen nahezu die Form von Biographien haben ${ }^{6}$, und andrerseits einige Biographien geradezu als Lehren bezeichnet werden (die Biographien des $S h t p-j b-R^{c}$ aus der 12. Dy-

\footnotetext{
3 Vernus, Sagesses, 23.
}

${ }^{4}$ Vgl. etwa H. Brunner in: Studien zu Lebenslehren, 169; id. in: A. B. Lloyd (ed.), Studies in Pharaonic Religion and Society in Honour of J. Gwyn Griffiths, London 1992, 164-8; J. Bergman in: Studien zu Lebenslehren, 86-104; E. Otto, HdO I,1,2, ${ }^{2} 1970$, p. 182; P. Vernus, Sagesses, $22-4$.

${ }^{5}$ Gnirs, Autobiographie, 208. Ähnlich A. Loprieno in: A. Loprieno (ed.), Ancient Egyptian Literature, PÄ 10, 1996, 404-5; Vernus, Sagesses, 23; s. u., $₫ 8$.

Brunner, Weisheit, 83; Bergman in: Studien zu Lebenslehren, 87; Vernus, Sagesses, 23. 
nastie und die des Jmn-m- h3t aus der 18. $)^{7}$. Umgekehrt ist aber auch hervorgehoben worden, $\mathrm{da} \beta$ sich in den Biographien insgesamt doch auffallend wenig unmittelbare Übernahmen aus den Lehren, wörtliche Zitate, ausmachen lassen: „Es fällt geradezu auf, wie wenig Anleihen bei den Lehren gemacht werden, wie selten also Zitate sind ${ }^{8}$." „Diese meist kurzen prägnanten Sätze [der Idealbiographen] stimmen inhaltlich (nicht wörtlich) mit den Weisheitslehren überein" " Dennoch wird generell eine enge innere Beziehung beider Textarten vermutet.

Es gibt aber auch gravierende Unterschiede, die selbst bei einem nur flüchtigen Vergleich beider Gattungen sogleich auffallen: Es ist eben nicht so, daß die Lehren eher allgemeine Richtlinien vermitteln und die Biographien dann deren Umsetzung ins konkrete Detail schildern, häufig hat man fast einen entgegengesetzten Eindruck: Die in den Lehren vermittelten Verhaltensregeln betreffen meist spezifische Situationen, die Biographien schildern die Handlungen des Protagonisten dagegen oft ohne jede situative Einschränkung. Und auch sonst gibt es vielfältige äußere und innere Unterschiede. Ein eingehenderer Vergleich beider Textsorten sollte also aufschlußreich sein.

2. Zuvor ist aber festzustellen, was eigentlich im einzelnen verglichen werden soll. Unter Lebenslehren werden hier - wie allgemein üblich diejenigen Texte verstanden, die in Form einer Rede des Lehrenden an den Schüler (bzw. in der literarischen Fiktion der Vater an den Sohn) Anweisungen und Ratschläge zum richtigen und erfolgreichen Verhalten in Beruf und Privatleben geben. Das sind im einzelnen folgende Texte: die Lehren des Djedefhor, Ptahhotep, Kagemni, Cheti, die Loyalistische Lehre, die Lehre

Kairo CG 20538 bzw. Urk IV, 1408-11; vgl. Brunner, Weisheit, 84; id., Fs Griffiths (s. Fußnote 4), 165; Bergman in: Studien zu Lebenslehren, 93; Gnirs, Autobiographie, 208-9; B.van de Walle, LÄ I, 819.

${ }^{8}$ Brunner in: Studien zu Lebenslehren, 169. Als Grund dafür vermutet Brunner, offenbar hätten „die Autobiographien eine andere Entstehung als die aus einer Umsetzung der Lehren" gehabt (loc. cit.).

${ }^{9}$ E. Otto in: HdO I, 1,2, ${ }^{2} 1970,182$. eines Mannes für seinen Sohn, die Lehren des Ani, Amunnacht, Amenemope, Papyrus Chester Beatty IV, Papyrus Brooklyn 47.218.135, Anchscheschonqi, der Papyrus Insinger, der Papyrus Louvre 2914 und einige Fragmente ${ }^{10}$. Üblicherweise werden auch die Lehre des Amenemhet und die Lehre für Merikare hinzugenommen, aber diese Texte gehören in die königliche Sphäre (vor allem an den jungen König gerichtet) und haben dadurch eine besondere Stellung. Sie können nicht ohne weiteres herangezogen und mit den (grundsätzlich nichtköniglichen) Biographien verglichen werden.

Unter einer (ägyptischen) (Auto)Biographie wird hier die in einer Inschrift festgehaltene zurückblickende Darstellung des Lebens einer realen Person zum Zweck der personalen Unsterblichkeit verstanden. Eine extensionale Definition wie bei den Lehren ist bei dieser Textsorte nicht sinnvoll.

3. Die Lehren gehören nach Thema und Verwendungssituation zum Diesseits, zur Alltagswelt, zur profanen menschlichen Sphäre. In ihnen wendet sich eine Lehrautorität an einen Schüler (d.h. ein älterer Beamter an einen Schreiberlehrling) zum Zweck der beruflichen und gesellschaftlichen Erziehung (wobei literarische Fiktion und Wirklichkeit zumindest grundsätzlich übereinstimmen). Lehren sind auf Papyri, Ostraka oder Schreibtafeln überliefert, also auf Gegenständen, die ausschließlich als Textträger fungieren, und sie sind normalerweise in der jeweils gängigen Kursivschrift (Hieratisch oder Demotisch) geschrieben. Wenn einmal eine Lehre in Teilen auf einer Stele belegt ist ${ }^{11}$, handelt es sich zweifellos um eine sekundäre Überlieferungssituation. Lehren haben mithin keine innere Bindung an ihre Textträger oder an den Ort, wo sie aufgezeichnet sind, der Text selbst gibt alle zum Verständnis notwendigen Erläuterungen.

${ }^{10}$ Brunner, Weisheit, Nr. 9; 11; Vernus, Sagesses, Nr. $9 ; 10 ; 14 ; 15$.

${ }_{11}$ Die Loyalistische Lehre auf der Stele Kairo CG 20538 aus Abydos. 
Die Biographien sind Teil der funerären bzw. sakralen Sphäre ${ }^{12}$. In ihnen spricht ein Toter zur Nachwelt zum Zweck der Erhaltung des personalen Gedächtnisses und zur Aufrechterhaltung des Totenkults. Die ersten Biographien waren Grabinschriften, und im funerären Bereich sind sie bis in späteste Zeit belegt, auf Grabwänden, Stelen, Grabstatuen, selten (spät) auch auf Sarkophagen und anderen funerären Objekten. $\mathrm{Ab}$ der 1. Zwischenzeit finden sie sich auch auf Felswänden (Felsinschrift bzw. „Felsstele“), und ab dem Neuen Reich kommen sie häufig im Tempel vor, vor allem auf Tempelstatuen, selten auch auf Tempelwänden. In allen Fällen handelt es sich sozusagen um „monumentale Privatinschriften", in Hieroglyphen geschrieben ${ }^{13}$. Biographien sind deutlich durch ihre „Umgebung“ determiniert: ihren Textträger, den Ort, an dem sie aufgezeichnet sind, und die spezifische Situation, für die sie konzipiert sind. Denn die fortdauernde Erinnerung an eine Person, die eine ägyptische Biographie erreichen soll, erstreckt sich ja nicht auf die gesamte Gesellschaft oder gar auf die ganze Welt, sondern nur auf die unmittelbare Umgebung: Familie, Kollegen, Graboder Tempelbesucher und deren Nachkommen. Diese Art der „Unsterblichkeit“ ist unlösbar mit dem Totenkult für eine Person verbunden, sein Fortbestehen ist der entscheidende Punkt. Darum legen viele Biographien das Schwergewicht nicht auf historisch bedeutsame Taten, die vielleicht aus sich heraus eine Erinnerung rechtfertigten, sondern sie zeigen, daß dieser Tote sich so verhalten hat, daß er eines fortdauernden Totenkults und der damit verbundenen Erinnerung würdig ist. Daher ist ein biographischer Text ohne seine situative Einbettung (und ohne die andersartigen, nichtbiographischen Textsorten, mit denen zusammen er meist vorkommt) zwar verständlich und sinnvoll, sein eigentlicher Zweck würde so aber nicht recht deutlich.

${ }^{12}$ Das gilt natürlich auch für die auf Felswänden aufgezeichneten Biographien, denn auch diese Orte sind ja durch die Beschriftung gewissermaßen zu „Gedenkstätten" geworden (oft auch durch bildliche Darstellungen verdeutlicht).

In der Spätzeit gibt es ganz vereinzelt auch demotische „Biographien“, vgl. J. Ray, JEA 73, 1987, 16980; JEA 75, 1989, 243-4.
4. Auch Form und Struktur von Lehren und Biographien sind sehr verschieden. Zunächst sind die Lehren für ägyptische Verhältnisse grundsätzlich „Langformen", die Biographien „Kurzformen“: Die Lebenslehren haben immer eine Länge von mehreren Papyrusseiten, was jeweils ein abgeschlossenes „Buch“ ergibt. Die Länge biographischer Texte ist sehr unterschiedlich. Sie können nur wenige Worte umfassen, aber auch eine ganze Stele oder Grabwand ausfüllen. Die Länge eines (ägyptischen) Buchs erreichen sie allerdings nie.

Lehren sind im allgemeinen selbständige, in sich abgeschlossene Texte und grundsätzlich auch als solche überliefert. Sie werden nicht in andere Textsorten eingebettet und schließen auch keine anderen in sich ein. Die Biographien können selbständige Texte $\operatorname{sein}^{14}$, in aller Regel kommen sie aber zusammen mit nichtbiographischen Textteilen vor, v. a. den „Anrufen an die Lebenden“, häufig auch mit Sicherungsformeln, Opferformeln, Gebeten, Fürbitten, Verklärungen u. a. m. In solchen aus Elementen mehrerer Textsorten zusammengesetzten Kompositionen haben die biographischen Passagen neben ihrer eigentlichen Aussage auch eine innertextliche Funktion: die Berechtigung einer Bitte um Opfer und Weiterführung des Totenkults nachzuweisen. Daher gibt es „Biographien“, die nur aus wenigen Worten bestehen (z. B. „denn ich war ein Gerechter, dem man opfern soll“), aber sie können der Länge wie dem Interesse nach auch das Zentrum einer solchen Komposition bilden. In jedem Fall ist die Biographie im Gegensatz zu den Lehren eine Textsorte, die oft in andere Textsorten eingebettet vorkommt und ebenso andere in sich einschließen kann. Darum wäre es in den meisten Fällen eigentlich richtiger, nicht von „(Auto)Biographien“ (im Sinne von eigenständigen Texten) zu sprechen, sondern von „(auto)biographischen Äußerungen“.

Lebenslehren haben - soweit der Anfang erhalten ist - eine Überschrift und, darin enthalten, eine Eigenbezeichnung, eben „Lehre“ (sb3yt) (obwohl die Bezeichnung „Lehre“ nicht

${ }^{14}$ Z. B. die Biographie des Ahmose von El-Kab, Urk IV, 1-11. 
nur die Lebenslehren umfaßt, sondern auch anderes, $z$. B. theologische Traktate). Neben der Überschrift können sie auch weitere Textelemente enthalten: Mehrere Lehren (Ptahhotep, Kagemni, Cheti, Anchscheschonqi) haben eine Rahmenerzählung, und auch ein Abschnitt über den Nutzen der Lehre findet sich öfter, meist als Epilog (Ptahhotep, Cheti, Amenemope). Alle diese Elemente haben aber gewissermaßen nur Hilfsfunktion für den Kern des Textes: die Belehrung selbst. Sie zeigen, wie sie zustandekam, wer sie erteilt, welchen Nutzen sie hat. Der Text der eigentlichen Lehre bespricht die einzelnen Themen, über die der Schüler belehrt wird, meist in ziemlich bunter Reihenfolge, die am ehesten durch assoziative Verknüpfungen zu erklären sein dürfte ${ }^{15}$. Nur die späteste Lehre des Papyrus Insinger faßt die verschiedenen Themen in eigenen Kapiteln zusammen.

Für die Biographie gibt es - wie für die meisten Textsorten - keine ägyptische Bezeichnung. Allerdings finden sich hin und wieder in den Anrufen an die Lebenden sozusagen „metatextliche" Verweise auf eine folgende oder vorhergehende biographische Passage, z. B. „dies alles, was ich auf Erden getan habe, was eingraviert ist auf [dieser] Statue ${ }^{616}$. Meist sind entsprechende Verweise mit einem "Anruf an die Lebenden“ verbunden: „Ich spreche zu euch, alle Menschen, ich lasse euch wissen die Gunstbeweise, die mir zuteil wurden “ ${ }^{\text {17 }}$, ,ich habe dies gesagt, was ich euch gesagt habe, damit es jedermann sehen kann “" ${ }^{18}$, „,ich lasse euch mein Wesen wissen (zu der Zeit), als ich auf Erden war ${ }^{\text {( }{ }^{19}}$ oder „wer von euch zu jung sein sollte für diese Rede, frage deinen Vater, er wird (es) dir sagen ${ }^{66^{20}}$ u. ä. Auch Beteuerungen, daß man seine Aussagen nicht erfunden oder übertrieben hat, können einen biographischen Text beginnen ${ }^{21}$, abschlie-

\footnotetext{
5 Vgl. Quack, Ani, 63-65.

${ }^{16}$ Jansen-Winkeln, Ägypt. Biogr., $472(h, 9)$.

Urk IV, 1,17-2,1; ähnlich 30,12-13.

Urk IV, 65, 10-11.

KRI III, 298, 2; JEA 70, 1984, 70, Z. 1-2.

${ }^{20}$ Urk I, 78,1-3; vgl. M. Lichtheim, Ancient Egyptian Autobiographies Chiefly of the Middle Kingdom, OBO 84, 1988, 23-4, n. 6.

Z. B. Siut V, 1-2.
}

$\operatorname{Ben}^{22}$ oder in ihn eingeschoben $\operatorname{sein}^{23}$. Schon im Alten Reich ${ }^{24}$ und in der Ersten Zwischenzeit ${ }^{25}$ versichert man, daß die biographische Äußerung wahr ist und keine bloße Pflichtübung der Grabinschrift („Amt der Nekropole“). Deutliche Gliederungen finden sich vor allem in denjenigen Biographien, die selbständige Texte bilden. So beginnt die Inschrift des Ahmose von El$\mathrm{Kab}^{26}$ mit einer Anrede an den Leser und einer kurzen Zusammenfassung des Inhalts, dann folgen chronologisch geordnet seine Kriegstaten und Belohnungen, dann eine abschließende Bemerkung über Alter und Anlage des Grabes sowie eine Liste der Belohnungen. Überhaupt stellt die Laufbahnbiographie das Leben des Protagonisten in der Regel implizit oder expli$\mathrm{zit}^{27}$ in seinem chronologischen Ablauf dar (zumindest grob), wenn das auch keineswegs zwingend so ist ${ }^{28}$. Dagegen sind idealbiographische Passagen ihrer Natur entsprechend nicht chronologisch gegliedert, und ihre thematische Gliederung ist oft schwer durchschaubar. Phrasen gleichen und ähnlichen Inhalts werden oft an unterschiedlichen Stellen wiederholt ${ }^{29}$. Ähnlich ist es dort, wo nicht die eigentliche Laufbahn geschildert, sondern nur das enge Verhältnis zum König thematisiert wird ${ }^{30}$.

Die Lehren sind - abgesehen von einigen narrativen Elementen in den Rahmen,,erzäh-

${ }^{22}$ Urk IV, 429, 16-430,1.

${ }^{23}$ Z. B. Urk IV, 438,16-439, 2; 973,8-13.

Z. B. Urk I, 267,3-4.

25 Z. B. Vandier, Mo alla, $242(\mathrm{~V}, \gamma, 2-3)$.

${ }^{26}$ Urk IV, 1-11.

${ }^{27}$ Z. B. Urk I, 215-221; Urk IV, 1-11; KRI III, 297-99.

${ }^{28}$ Vor allem die Priesterbiographien der Spätzeit sind oft in keiner Weise chronologisch geordnet.

${ }^{29} \mathrm{Vgl}$. etwa die biographischen Passagen auf London BM 581 (Sethe, Lesestücke, 80-81); BM 572 (BM Stelae, II, pl. 22); New York, MMA 57.95 (Fischer, JNES 19, 1960, 258-68; pl. VII); Kairo CG 20543 (H. O. Lange/H. Schäfer, Grab- und Denksteine des Mittleren Reiches, II, Berlin 1908, 164-7) und sonst oft. Möglich wäre eine assoziative Verknüpfung, wie das bei den Lehren wahrscheinlich ist (s. o.). Bei den Biographien ist aber auch zu berücksichtigen, daß sie in vielen Fällen phraseologisch von älteren Vorbildern abhängig sind und vielleicht deren Reihenfolge in Teilen einfach übernommen haben.

Z. B. auf London BM 614 (TPPI, 15-17) und sonst oft. 
lungen" (die aber auch zum Teil aus wörtlichen Reden bestehen können) - in der Textform "Rede" gehalten" ${ }^{31}$. Die eigentliche Lehre ist sehr einfach strukturiert: sie besteht aus Aufforderungen (ein nicht explizit bezeichneter Sprecher redet einen in der 2. Person Singular bezeichneten Hörer an) und allgemeingültigen, konstatierenden Aussagen, die die Aufforderungen begründen, z. B.

Ptahhotep 52-7: „Sei nicht eingebildet auf dein Wissen, und vertraue nicht darauf, daß du ein Gelehrter bist ... Es gibt keinen Kundigen, dessen Wirken vollkommen ist, und die Grenzen der Kunst werden nie erreicht ${ }^{32}$."

Ptahhotep 441/446-7: „Beuge deinen Rücken vor deinem Vorgesetzten ... schlecht fährt, wer seinem Vorgesetzten widerstrebt; man lebt, wenn er gnädig ist ${ }^{33}$.

Amenemope 5,10-15: „Zettele keinen Streit an mit dem Heißmäuligen und stichle ihn nicht mit Worten ... ein Sturmwind, der losbricht wie Feuer im Stroh, das ist der Hitzige in seiner Stunde ${ }^{34}$."

Amenemope 14,5-8: „Sei nicht begehrlich nach der Habe eines Armen und nicht hungrig nach seinem Brot. Die Habe eines Armen bleibt in der Kehle stecken und läßt den Hals erbrechen ${ }^{4{ }^{435}}$.

Biographien können in der Textform „Bericht" gehalten sein (nur Laufbahnbiographien, z. B. des Ahmose ${ }^{36}$ oder des Amenemhab ${ }^{37}$ ), in aller Regel sind aber auch sie „Reden“. Dabei ist die Grundform eine konstatierende Rede in der 1. Person mit nicht explizit bezeichnetem Hörer. Diese Grundform kann aber je nach Rahmentext variieren: Bei einer in ein Gebet eingebetteten Biographie haben wir oft die Konstellation

\footnotetext{
${ }^{31} \mathrm{Zu}$ den „Textformen“ („Rede“ und „Bericht") s. K. Jansen-Winkeln, Text und Sprache in der 3. Zwischenzeit, ÄUAT 26, 1994, 12-16; J. Winand, Lingua Aegyptia 6, 1999, 220-23 (der eine andere Terminologie bevorzugt) id, BiOr 52, 1995, 311-3. 20.

Z. Zába, Les maximes de Ptahhotep, Prag 1956, Ibid., 51.

H. O. Lange, Das Weisheitsbuch des Amenemope, Kopenhagen 1925, 39-40.

Ibid., 71.

Urk IV, 1-11.

${ }^{37}$ Urk IV, 890-97.
}

1.-2. (Gott angeredet) $)^{38}$, bei einer in eine Fürbitte eingebetteten dagegen 1.-3. (Rede in der 1. Person über eine 3. Person). Diese letztere Konstellation findet sich öfter auf gestifteten Objekten: Der Sohn läßt den Vater über sich sprechen $^{39}$. Bei einer in eine Verklärung eingebetteten Biographie ist die Rede (anonym) an eine 2. Person gerichtet (die eigene Biographie wird ihr erzählts) ${ }^{40}$ etc. Der Begriff Autobiographie wäre also zu eng. Besonders charakteristisch für Biographien (in jeder Redekonstellation) sind eulogische Passagen mit Reihungen von Beiwörtern, oft von beträchtlicher Länge. Sie können aus bloßen attributiven bzw. appositionellen Ausdrücken bestehen, meist in die Titelreihe eingeschoben, oder als Nominalsätze auftreten (jnk A, B, C ...). Die Lehren sind klar getrennt in solche, die an eine königliche Person gerichtet sind (hier nur am Rande berücksichtigt), und solche, die sich an den Beamtennachwuchs wenden. Sie unterscheiden sich nicht nur in ihren (innertextlichen) Adressaten, sondern auch nach Themen und Sprachform. „Historische Rückblicke" kommen nur in den königlichen Lehren vor, ebenso autobiographische Passagen $^{41}$. Die Unterschiede sind so deutlich, daß die „Königslehren“ und die anderen Lebenslehren kaum zur gleichen Textsorte zu rechnen sind. Innerhalb der „eigentlichen“ Lehren bilden diejenigen des Mittleren Reiches, die vor allem Loyalität zum König fordern (die Loyalistische Lehre, die Lehre eines Mannes für seinen Sohn), einen eigenen Typus, ebenso vielleicht die Lehre des Cheti mit ihrer starken Berufsbezogenheit. Die anderen lassen sich kaum

${ }^{38}$ Vgl. etwa Kairo CG 42208, c, s. JansenWinkeln, Ägypt. Biogr., 47-49; 454-6 (und sonst oft, v. a. in der Spätzeit).

Z. B. auf Kairo CG 42220, s. Jansen-Winkeln, Ägypt. Biogr., 279-80.

Z. B. Petosiris, Inschriften Nr. 58 und 61, s. G. Lefebvre, Le tombeau de Petosiris, Bd. 2, Kairo 1923, 29-32; 35-38.

${ }^{41}$ In den anderen Lehren eigentlich nur in den "Rahmentexten“ als Äußerungen des Lehrers, vgl. etwa Cheti, IV, 1-7 (W. Helck, Die Lehre des Dw3-Htjj, I, Wiesbaden 1970, 19-35), pChester Beatty IV, vso., 6,3-4 (pHier. BM [Gardiner], 43; pl. 20), pInsinger, 35, 2-5 (F. Lexa, Papyrus Insinger, I, 1926, 111-2). 
weiter in Typen unterteilen, und es sind ja auch nicht sehr viele Texte. Die Biographien werden meist grob in "Laufbahnbiographie“ (bzw. „Ereignisbiographie ${ }^{\text {"42 }}$ ) und „Idealbiographie“ eingeteilt, die ersteren berufliche Erfolge und sonstige bemerkenswerte Ereignisse, die letzteren die persönlichen Werte referierend. A. Gnirs unterteilt die Laufbahnbiographie weiter in „Ereignis-“ und „Handlungsbiographie“ und möchte die Idealbiographie in "Reflexionsbiographie“ umbenennen ${ }^{43}$. Daneben setzt sie noch für den Sonderfall der ramessidischen Sündenbekenntnisse eine „Bekenntnisbiographie“ an ${ }^{44}$. Die verschiedenen Typen von Biographie sind im übrigen keineswegs streng getrennt, sondern gehen verschiedentlich ineinander über. Vor allem aber gehören in ein und demselben Text oft bestimmte Passagen zum Typ Ereignisbiographie und andere zum Typ Idealbiographie.

Sprachlich sind Lehren und Biographien zunächst unterschiedslos alt- bzw. mittelägyptisch abgefaßt. Nach Verschriftung der jüngeren Sprachstufe ab der Amarnazeit trennen sich jedoch die Wege: Die Lebenslehren sind grundsätzlich in der jüngeren Sprachstufe gehalten (neuägyptisch, später demotisch), die Biographien in der älteren (spätmittelägyptisch). Das liegt daran, daß die Lehren zur profanen Sphäre (s. o., \3) gehören, für die ab der 19. Dynastie zunehmend das Neuägyptische verwendet wird, die Biographien dagegen zur funerären und sakralen Sphäre, in der man die ältere Sprachstufe beibehält. Auf diese Weise ergibt sich ab dem späteren Neuen Reich eine zusätzliche divergierende Entwicklung beider Textsorten, und ihre Zugehörigkeit zu den verschiedenen Welten des

${ }^{2}$ N. Kloth, Die (auto-)biographischen Inschriften des ägyptischen Alten Reiches: Untersuchungen zu Phraseologie und Entwicklung, SAK, Beihefte, 8, 2002, 229 , weist zurecht darauf hin, daß die „Laufbahnbiographie“ eigentlich nur eine Untergruppe der „Ereignisbiographie" ist.

${ }^{43}$ Gnirs, Autobiographie, 204. Der Terminus „Idealbiographie“ wird oft als unangemessen gerügt.

${ }^{44}$ Eine weitere von ihr angesetzte Unterart, die „,enkomiastische Autobiographie“, wird mir nicht recht deutlich; es ist jedenfalls kein Typ von Biographie, der gleichberechtigt neben Ereignisbiographie und Idealbiographie steht.
Diesseits und Jenseits wird nun auch in ihrer Sprache deutlich.

Ein weiterer Unterschied, der von Anfang an bestand, ergibt sich aus dem ,literarischen" Charakter der Lehren: Es waren Texte, die von vielen genutzt und kopiert wurden. Sie wurden im Unterricht verwendet, und zwar nicht nur in ihrer Entstehungszeit, sondern noch Jahrhunderte später, als sie „Klassiker“ geworden waren und dem mittelägyptischen Sprachunterricht dienten ${ }^{45}$. Viele Lehren sind in den Rang ,,kultureller Texte" erhoben worden ${ }^{46}$, wie sich nicht nur aus ihrer vielfältigen Bezeugung ergibt, sondern auch aus der Aufzählung klassischer Schriftsteller im Papyrus Chester Beatty IV. Für die Biographien gilt das alles nicht. Es sind eben keine Texte, die sich durch ihre individuelle Gestaltung und Einzigartigkeit abheben sollen, vielmehr verwendet man hier - vor allem in der Idealbiographie - gern feststehende und nur geringfügig variierte Phrasen, die über Jahrhunderte tradiert worden sind. Man ordnet sich auf diese Weise bewußt in eine lange (und anonyme) Tradition ein.

5. In vielen, nahezu allen Bereichen unterscheiden sich Lebenslehren und Biographien also deutlich: in der Verwendungssituation, der formalen Struktur, dem literarischen Charakter und ab dem späteren Neuen Reich sogar in der Sprache. Eine nähere Verwandtschaft sollte man bei den Inhalten erwarten.

Die Themen der Lebenslehren sind vor allem das Verhalten in Beruf, Gesellschaft und Familie: wie man mit Vorgesetzten umgeht oder mit gleichrangigen und untergeordneten Kollegen, wie man berufliche Aufträge erledigt, wie man sich bei Tisch oder als Gast benimmt, wie man bei verschiedenen Anlässen redet, wie man sich gegenüber den Armen verhält etc. Das sind die allgemeinen Themen, die in fast allen Lehren vorkommen. Daneben gibt es auch einige spezifischere, die auf bestimmte Texte beschränkt sind: So werden in der Lehre des Cheti ausgiebig die Vorzüge des Schreiberberufs und die Nach-

${ }^{45}$ S. J. Assmann in: ZDMG, Suppl. VI, 1985, $43 \mathrm{ff}$.

${ }^{6}$ Vgl. dazu Assmann in: A. Loprieno (ed.), Ancient Egyptian Literature, PÄ 10, 1996, 59-82. 
teile aller anderen Tätigkeiten hervorgehoben (ähnlich auch im pChester Beatty IV), in der Loyalistischen Lehre und der Lehre eines Mannes für seinen Sohn geht es in erster Linie um die Notwendigkeit der Loyalität zum König. Die königlichen Lehren (Lehre des Amenemhet, Merikare) fallen naturgemäß fast ganz aus diesem Rahmen, weil die Pflichten eines Königs anderer Art sind als die eines Beamten. Immerhin gibt es auch hier durchaus noch Gemeinsames, etwa die Ausführungen über die Vorzüge der Beredsamkeit, des vorausschauenden Handelns, der Gerechtigkeit.

Die bevorzugten Themen der Biographien variieren noch stärker, je nachdem, wer ihr Protagonist ist und in welcher Epoche sie verfaßt wurden. Im Alten Reich etwa steht nahezu ausschließlich der König und das Verhalten des jeweiligen Beamten zu ihm im Mittelpunkt; die Laufbahn des Beamten wird als Folge königlicher Gnadenerweise dargestellt. Insgesamt gesehen sind aber während ihrer gesamten Überlieferungszeit bestimmte Themenkreise besonders typisch für die Biographie:

\section{Das Verhältnis zum König;}

2. allgemeines Verhalten im Beruf (Tätigkeit für Vorgesetzte, gutes Verhalten zu Untergebenen, Lösung von Problemen, Übertreffen früherer Leistungen);

3. spezifische berufliche Tätigkeiten (Verwaltung, Rechtsprechung, Bauwesen, Militärisches, Tempelverwaltung);

4. soziale Stellung und Verhalten zu Mitmenschen (Ansehen, Umgänglichkeit, Wohltätigkeit, Diskretion);

5. menschliche Eigenschaften (Charakter, Klugheit, Beredsamkeit, Schweigen, Beherrschtheit);

6. Religiöses (v. a. Frömmigkeit und kultische/ priesterliche Tätigkeiten);

7. Familiäres (Kindheit, Eltern, Abstammung, Amtsnachfolge des Vaters, Alter, Krankheit, Tod) und Sonstiges aus dem Privatleben (Feste feiern, Heimatverbundenheit etc.).

Wenn man die Themenspektren von Lehren und Biographien vergleicht, fällt sogleich auf, daß ganze Komplexe nur in den Biographien, nicht aber in den Lehren vorkommen, und zwar vor allem drei Themenkreise:
1. Das Verhältnis zum König: der Umgang mit ihm, die Stellung bei Hof, die Förderung durch den König, Tätigkeiten in seinem Auftrag. Dies sind in den Biographien prominente Themen (nicht in allen, aber doch in sehr vielen, vom Alten Reich bis zur Spätzeit). In den Lehren kommt dagegen nahezu nichts aus diesem Bereich vor. Der König wird überhaupt nur selten erwähnt, vorwiegend in zwei Texten, der Loyalistischen Lehre und der Lehre eines Mannes für seinen Sohn, aber dort auch nur, um zur Treue zum König aufzufordern und seine Macht $\mathrm{zu}$ feiern ${ }^{47}$.

2. Auch die in Biographien häufigen Angaben zu spezifischen beruflichen Tätigkeiten haben in der Lehre verständlicherweise keine Entsprechung. Anders etwa als bestimmte Schultexte (z. B. der pAnastasi I), in denen ganz konkrete fachspezifische Probleme mathematischer und technischer Art beschrieben werden (und deren Vokabular die Schüler auf diese Weise lernten), befassen sich die Lebenslehren nur mit den allgemein menschlichen Seiten des Berufslebens, wie etwa dem Verhalten gegenüber Vorgesetzten. Auch beim Lob des Schreiberberufes geht es nicht um eine besondere Tätigkeit, sondern um die Überlegenheit des Schreibers als solchem, gleichgültig in welcher spezifischen Tätigkeit.

3. Der dritte größere Themenkreis, der in den Biographien von Bedeutung ist und sogar immer bedeutender wird, in den Lebenslehren dagegen verhältnismäßig selten angesprochen wird, ist Religion und Frömmigkeit. In den Biographien ist dieser Komplex immer von Bedeutung gewesen, er nimmt im Verlauf der Zeit noch an Umfang zu, und in der Spätzeit ist er mit Abstand das häufigste Thema überhaupt. Darunter zu verstehen sind Aussagen darüber, daß der Tote besonders fromm und gottesfürchtig war, von Gott geliebt und begünstigt wurde und ihm Priesterdienste leistete. Gerade diese kultischen Tätigkeiten werden oft in großer Ausführlichkeit entwickelt. Dazu gehören auch Dinge, die eher in den Bereich der Tempelver-

${ }^{47}$ In der Lehre des Anchscheschonqi (25, 10-11) soll der Schreiber auch lernen, wie man an den König schreibt und sich in seiner Gegenwart verhält. 
waltung gehören, wie etwa Bau und Restaurierung von Kapellen, ihre Versorgung mit Geräten und Gütern usw.

In den Lehren sieht es damit ganz anders aus: Es finden sich nur vereinzelt Sätze, in denen vergleichbare Dinge angesprochen wurden, z. B. im Djedefhor die Aufforderung, ein Grab anzu$\operatorname{legen}^{48}$ (ebenso im Anchscheschonqi ${ }^{49}$ und im $A i^{50}$ ) und für den Totenkult zu sorgen, in der Lehre des Ani, die religiösen Feste zu begehen ${ }^{51}$, den Totenkult der Eltern zu erhalten ${ }^{52}$, Ehrfurcht vor Gott zu haben ${ }^{53}$, in pChester Beatty IV zu opfern ${ }^{54}$ und Gottes Besitz zu achten ${ }^{55}$. Etwas häufiger wird Gott im Anchscheschonqi, im Insinger und in der Lehre des Amenemope erwähnt, aber auch in dieser letzteren geht es nicht in erster Linie um Fragen der Religion, der Frömmigkeit oder des Kults. Vielmehr dient Gott in der Lehre v. a. der Begründung der Handlungsanweisungen: Ein direkter Zusammenhang zwischen rechtem Verhalten und erfolgreichem Leben kann nicht guten Gewissens behauptet werden, daher dient Gottes Wille und die Ergebung in seine unerforschliche Macht als Begründung, dieses rechte Verhalten zu empfehlen.

Von den sieben Themenkreisen, die in den Biographien oft angesprochen werden, sind also nur vier in den Lehren ebenfalls prominent vertreten: das allgemeine Verhalten im Beruf, Stellung und soziales Verhalten, menschliche Eigenschaften, Familie und Privatleben.

${ }^{48}$ W. Helck, Die Lehre des Djedefhor und die Lehre eines Vaters an seinen Sohn, Wiesbaden 1984, 12-14; Brunner, Weisheit, 103; Vernus, Sagesses, $49-50$.

49 12,5, s. Thissen, Die Lehre des Anchscheschonqi, 25.

50 17,14-17, s. Quack, Ani, 96/97; 291; Vernus, Sagesses, 245.

${ }_{51}$ 16,3-4, s. Quack, Ani, 90/91; 285; Vernus, Sagesses, 243.

52 17,4-6, s. Quack, Ani, 94/95; 289-90; Vernus, Sagesses, 244.

20,12-15, s. Quack, Ani, 108/109; 311-12; Vernus, Sagesses, 249-50.

${ }^{54}$ Vso., 4,10-12, s. pHier. BM (Gardiner), 42; pl. 19; Brunner, Weisheit, 227-8; Vernus, Sagesses, 275-6.

${ }^{55}$ Vso., 5,2-4, s. pHier. BM (Gardiner), 42; pl. 20; Brunner, Weisheit, 228; Vernus, Sagesses, 276.
Umgekehrt gibt es auch einige Themen, die zwar in den Lehren recht häufig sind, aber in den Biographien so gut wie nie auftauchen. Das sind vor allem Dinge aus dem privaten Bereich, z. B. Belehrungen darüber, wann man sich eine Frau nehmen soll und wie man sich zu ihr verhält. Aussagen zu Frauen und zum Verhältnis von Mann und Frau sind in den Lehren durchaus üblich, in den Biographien dagegen fehlen sie völlig; die Ehe(frau) ist dort kein Thema ${ }^{56}$. Wenn überhaupt die Familie erwähnt wird, dann Vater, Mutter und Geschwister, fast stets in stereotypen Wendungen und wenig ausführlich. Man sagt allenfalls, daß in dieser Hinsicht alles zum besten stand (,geliebt von seinen Eltern, gelobt von seinen Brüdern" $)^{57}$. Dasselbe gilt von der Anlage eines eigenen Hauses und eines Gartens, die in den Lehren empfohlen wird: Auch davon schweigen die Biographien durchgehend, ebenso zu Themen wie dem Verhalten bei Tisch oder der notwendigen Mäßigung beim Trinken, beides prominente Sujets der Lebenslehren, aber in den Biographien kaum jemals erwähnt. In ihnen wird dagegen öfter umgekehrt die festliche Trinkfreudigkeit gepriesen, z. B. ,ich machte meine Tage mit Wein und Myrrhe festlich“, oder man empfiehlt sogar ,leide keinen Durst neben Bier ${ }^{658}$.

Ein Bereich aus dem Berufsleben, der in den Lehren häufig vorkommt, nicht aber in den Biographien, ist das Verhalten als Untergebener, etwa wie man sich zu wütenden Vorgesetzten verhält (nicht widersprechen; warten, bis er sich beruhigt hat), im Vorzimmer eines Mächtigen oder als Bote eines höheren Beamten.

Gemeinsame Themen von Lebenslehren und Biographien sind also nur folgende: allgemeines Verhalten im Beruf, soziales Verhalten, menschliche Eigenschaften und Familie (mit gewissen Einschränkungen). Aber auch diese Themenkreise werden in den beiden Textarten bei wei-

${ }^{56}$ In den (seltenen) Biographien von Frauen sind allerdings gerade umgekehrt Ehe und Familie eines der Hauptthemen.

Vgl. Kloth, Die (auto-)biographischen Inschriften (s. o., n. 42), 61-65.

${ }^{58}$ Kairo CG 42225, Jansen-Winkeln, Ägypt. Biogr., 122; 498, Z. 4; 10; vgl. allgemein Otto, Biogr. Inschr., 70-3. 
tem nicht in gleicher Weise behandelt: Das jeweils beschriebene bzw. empfohlene Verhalten unterscheidet sich deutlich.

In den Lehren hat etwa die Warnung zur Besonnenheit einen besonders großen Stellenwert. Man soll in jeder Lebenslage ruhig, besonnen und auf keinen Fall ,hitzig“" sein, aber auch den Umgang mit dem „Hitzigen“ nach Möglichkeit meiden ${ }^{59}$. Auch in den Schultexten ist dies ein beliebtes Thema ${ }^{60}$. In den Biographien wird natürlich auch das Ideal der Besonnenheit (und des

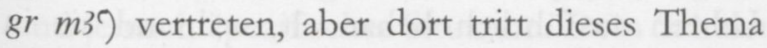
viel weniger hervor. Der "Hitzige“ wird nur selten erwähnt, und wenn, dann nicht etwa in dem Sinn, daß man ihm aus dem Weg ging, im Gegenteil, man stellte sich ihm entgegen und wehrte ihn $a b^{61}$.

Noch deutlicher ist der Unterschied bei dem nicht seltenen Topos der Lehren, was man zu tun hat, wenn man in einen Streit (anderer) zu geraten droht. Die Antwort ist dort stets die gleiche: aus dem Weg gehen, sich nicht einmischen $^{62}$. In den Biographien würde man solch ein Ausweichen vor einem Problem nie darstellen. Dort sagt man im Gegenteil, daß man schlichtend in einen Streit eingriff ${ }^{63}$ und zwei Streitende so versöhnte, daß beide zufrieden waren $^{64}$ (was im wirklichen Leben bekanntlich

Vgl. etwa Ptahhotep, 375-8, s. Žába, Les maximes de Ptahhotep, 46; Amenemope, Kap. 3; 4; 9; 10; Papyrus Insinger, Kap. 19 und 23.

Vgl. Fischer-Elfert, WdO 14, 1983, 83-90.

Z. B. Urk IV, 969.

${ }^{62}$ Vgl. etwa die Lehre des Cheti, 9,5-6 (s. Helck, Die Lehre des Dw3-Htjj, 125-6); die Lehre eines Mannes für seinen Sohn, \24 (s. H.-W. Fischer-Elfert, Die Lehre eines Mannes für seinen Sohn, ÄA 60, 1999, 248-53); Ani, 21, 16-18 und 19,15-16 (s. Quack, Ani, 114/115; 321-22; 104/105; 303-4); Amenemope, $5,10-12$ und $22,20-23,7$ (s. Lange, Das Weisheitsbuch des Amenemope, 40; 112-3); Anchscheschonki, 19,11-12 (s. Thiss en, Die Lehre des Anchscheschonqi, 32).

Z. B. auf der Stele BM 581: ,ich war jemand, der das Wort ergriff, wo man zornig war, der den Ausdruck kennt, worüber man in Wut gerät", s. Sethe, Lesestücke, 80 , Z. 17-18.

${ }^{64}$ S. z. B. Edel, MDAIK 13, 1944, 42-3 (\$ 37); Kloth, Die (auto-)biographischen Inschriften, 80-1; Schenkel, Memphis, Herakleopolis, Theben, 39; 1289; 149; Janssen, Autobiografie I, 53-4 (S); JansenWinkeln, Ägypt. Biogr., 341 (2.3.10). selten vorkommt). Anchtifi von $\mathrm{Mo}^{\mathrm{c}}$ alla (der gerne zugespitzt formuliert) behauptet sogar von seinen friedensstiftenden Bemühungen: „Ich bewirkte, daß ein Mann den Mörder seines Vaters und den Mörder seines Bruders umarm$\mathrm{te}^{\mathrm{6} 65}$. Besonders schön deutlich wird der Gegensatz zwischen den Empfehlungen der Lehren und den Behauptungen der Biographien auch in folgendem: In der Lehre eines Mannes für seinen Sohn wird geraten: „Trenne nicht zwei Männer in ihrer Wut; der Streit wendet sich gegen den, der ihn schlichten will" ${ }^{* 66}$. Dagegen sagt ein Offizier und Priester der 19. Dynastie in seiner Grabbiographie: „Ich war es, der zwei Männer trennte, als sie wütend waren, so daß sie in Frieden davon gingen ${ }^{6{ }^{67}}$.

Das Thema „Wohltätigkeit" nimmt in den Biographien traditionell großen Raum ein, vom Alten Reich bis in späteste Zeit ${ }^{68}$. Die Phrase ,ich gab Brot dem Hungrigen (Bier dem Durstigen) und Kleider dem Nackten " (u. ä.) ist sogar fast ein Schlüsselwort der Biographien, anhand dessen sich die Textsorte bestimmen ließe; solange es Biographien gibt, gibt es auch diese Phrase ${ }^{69}$. Dagegen spielt in den Lehren die Wohltätigkeit nur eine recht untergeordnete Rolle $^{70}$. In ihnen geht es eher darum, den Armen und Geringen nicht ungerecht $\mathrm{zu}$ behandeln oder zu schädigen, weniger darum, ihn aktiv zu unterstützen.

Gegensätzlich in Lehren und Biographien kann auch das Verhältnis zu den "Großen“ dargestellt werden. Die Lehren differenzieren sehr genau, wie man sich je nach Rang des Gegen-

${ }^{65}$ Vandier, Mo alla, 163 (I, $\left.\beta, 1\right)$.

$\int 24,3-4$ ( $m$ wpj z 2 m nšnj.sn wdb shwn $r$ wd $\left.d^{\ulcorner} s w\right)$, s. Fischer-Elfert, Die Lehre, 248-50.

${ }^{67}$ Grab des Anhurmose, Z. 48-9 (jnk wpj z 2 nšnjw $r$ [pr]y.st $m h t p w)$, s. B. Ockinga/Y.al-Masri, Two Ramesside Tombs at El Mashayikh, Part 1, Sydney 1988, pl. 27; 41-42.

${ }^{68} \mathrm{Vgl}$. etwa die bei J. Vandier, La famine dans l'Egypte ancienne, Kairo 1936, $99 \mathrm{ff}$. gesammelten (überwiegend biographischen) Texte.

Vgl. Edel, MDAIK 13, 1944, 40-1; Kloth, Die (auto-)biographischen Inschriften, 77-8; Janssen, Autobiografie I, 78-81; Otto, Biogr. Inschr., 94.

Vgl. etwa Ani 21,3-7 (Quack, Ani, 110/111; 317-8); pChester Beatty IV, vso., 1,13-2,5 (pHier. BM [Gardiner], 38; pl. 18); Papyrus Insinger, Kap. 15. 
übers verhalten soll; "Großen“ gegenüber auf jeden Fall demütig ${ }^{71}$. Ähnliches kann zwar auch in Biographien gesagt werden, z. B. „der den Arm beugt im Kreis der Großen" “72 oder ,ich ehrte die Großen “" ${ }^{67}$, viel öfter aber wird die bevorzugte Stellung des Sprechers unter den Großen betont (,,ich wurde größer gemacht als die Großen") ${ }^{74}$ oder seine Gerechtigkeit gegen Groß und Klein ${ }^{75}$. Ein Satz wie ,ich machte keinen Unterschied zwischen einem Großen und einem Geringen “6 ${ }^{676}$ läuft allen Empfehlungen der Lehren sogar direkt zuwider.

6. Die Beispiele für diese unterschiedlichen, manchmal geradezu entgegengesetzten Betrachtungsweisen ähnlicher Wechselfälle des Lebens in Lehre und Biographie ließen sich leicht vermehren. Die Gründe dafür ergeben sich aus dem jeweils anderen „Sitz im Leben“ dieser beiden Textsorten.

Der Umgang mit dem König spielt in den Lehren keine Rolle, weil sie der Erziehung des Schreibernachwuchses dienen, aus denen zunächst einmal kleine oder allenfalls mittlere Beamte werden sollten. Für diese Gruppe war an einen Umgang mit dem König gar nicht zu denken. Dagegen stammen Biographien überwiegend von hohen und höchsten Funktionären, die im Rückblick die Höhepunkte ihrer Laufbahn wiedergeben. Ebenso dienen die Lehren nicht der Ausbildung zu einem bestimmten Beruf, deshalb fehlen alle Angaben zu spezifischen beruflichen Tätigkeiten.

Auch bei dem Themenkreis „Religiöses“ ist der Grund für die Unterschiede zwischen Lehren und Biographien nicht schwer zu finden:

${ }^{71}$ Vgl. Ptahhotep, 175-85; 399-414; $441-47$ (s. Žába, Les maximes de Ptahhotep, 29-30; 48-9; 51; Brunner, Weisheit, 116; 124; 125-6; Vernus, Sagesses, 83; 98-99; 101); Ani, 19,10-12; 22,7-10 (Quack, Ani, 104/105; 302; 116-119; 327-29; Vernus, Sagesses, $248 ; 253-4)$.

${ }_{72}$ London BM 614, Z. 7, s. TPPI, 15.

${ }_{74}^{73}$ Jansen-Winkeln, Ägypt. Biogr., 368 (3.8.1).

${ }^{74}$ Schenkel, Memphis, Herakleopolis, Theben, 37; vgl. auch Janssen, Autobiografie I, 15-17; JansenWinkeln, Ägypt. Biogr., 369-71.

${ }^{75}$ Z. B. Siut III, 41, s. Schenkel, Memphis, Herakleopolis, Theben, 82 .

${ }^{76}$ Jansen-Winkeln, Ägypt. Biogr., 377 (3.10.23).
Die Biographien sind Texte der funerären und sakralen Sphäre, in Gräbern und Tempeln zu finden, sie sind ein Mittel zur Aufrechterhaltung des Totenkults für den Verstorbenen. Die Lehren sind demgegenüber profane Texte, an Leute gerichtet, die das Leben noch vor sich haben, und sie haben vor allem das Ziel, deren Verhalten im Alltagsleben zu beeinflussen. Deshalb werden auch bestimmte Privatangelegenheiten wie das Verhalten bei Tisch, die Gründung einer Familie oder das Verhältnis zur Ehefrau in den Lehren ausführlich behandelt, während sie in den Biographien so gut wie nie vorkommen. Dinge aus dem alltäglichen oder sogar intimen Bereich hielt man nicht für erwähnenswert, wenn es um das Nachleben einer Person ging ${ }^{77}$.

Das empfehlenswerte Verhalten gegenüber wütenden Vorgesetzten (den Kopf einziehen) erwähnen die Biographien schon deshalb nicht, weil in ihnen nur die Glanzpunkte des Lebens und der Laufbahn berichtet werden. Generell schildern sie nie das Ausweichen vor Problemen oder Umgehungsstrategien, alle Schwierigkeiten werden in ihnen glanzvoll gelöst.

Die meisten inhaltlichen Differenzen zwischen Lehre und Biographie ergeben sich daraus, daß die Lehre der Erziehung zum durchschnittlichen Verhalten dient, zur Mäßigung und Vorsicht, zu Alltag und Normalität. Die Welt wird aus der Perspektive des kleinen oder mittleren Funktionärs gesehen. Wichtig für diese Perspektive ist das genaue Differenzieren der Situationen und das Erkennen und Vermeiden von Problemen schon im Vorfeld. Die Biographien dagegen berichten die Höhepunkte des Lebens und die Laufbahnen von in der Regel hohen Funktionären, die aus der Menge herausragen in ihren Biographien vermutlich noch deutlicher als in ihrem wirklichen Leben. Daher geht es oft um Ausnahmesituationen, Kriegstaten, Expeditionen, Auszeichnungen. Die Welt wird aus der Perspektive des großen Herrn betrachtet und in der Rückschau berichtet.

Dieser fundamentale Unterschied prägt auch den Stil beider Textarten. Die Lehren differen-

Auch in modernen Autobiographien wird derartiges meist beiseitegelassen. 
zieren, ein Fall stellt sich in ihnen komplexer dar, und sie formulieren ihn umständlicher als die Biographien: „Wenn du ein fähiger Mann bist, der im Rat seines Herrn sitzt, konzentriere dich auf das Wesentliche. Sei schweigsam, das ist nützlicher als blumige Reden(?). Sprich (erst), wenn dir deine Lösung (des Problems) klargeworden ist. Nur der Sachkundige redet im Rat; Reden ist schwieriger als jede Arbeit. (Nur) wer sie versteht, macht sie sich dienstbar. ${ }^{6{ }^{78}}$ Sie begründen das richtige Verhalten psychologisch und je nach spezifischer Situation. Dem Schüler wird das Leben mit all seinen Unwägbarkeiten als etwas noch zu Bewältigendes vorgestellt.

Dagegen neigen die Biographien zum Vereinfachen, sie formulieren wesentlich, knapper und pointierter, nicht selten im Stile des ,veni, vidi, vici“: „Der frei redet, während die Edlen schweigen und das Volk still ist ${ }^{67^{79}}$. Alles Problematische wird ausgeblendet oder als gelöst beschrieben. Von dem in der Lehre des Ptahhotep geschilderten Verhalten im Vorzimmer (,Wenn du in einem Vorzimmer bist, dann stehe oder sitze, wie es dir zusteht, wie es dir vom ersten Tag an beigebracht worden ist; gehe nicht vorbei, sonst wirst du abgewiesen $\left.{ }^{6}\right)^{80}$ würde man in einer Biographie allenfalls das Ergebnis berichten: „Ich wurde nicht abgewiesen“. Häufiger aber klingt es so: „(Ich war) einer, der bei seinem Herrn [dem König] eintrat, während die Großen hinter ihm waren und die Türhüter sich beugten, bis ich den Platz erreicht hatte, wo seine Majestät war. ${ }^{\text {c }}{ }^{81}$

7. Die ägyptischen Lehren haben eine gut 2000jährige Geschichte, sie sind seit der 12 . Dynastie bezeugt. $\mathrm{Ob}$ einige der überlieferten Texte (etwa Ptahhotep und Hordjedef) wesentlich älter sind als diese Zeit, ist zumindest frag-

\footnotetext{
${ }^{78}$ Ptahhotep, 362-69, s. Žába, Les maximes de Ptahhotep, 33.

${ }^{79}$ Bersheh, II, pl. XIII, Z. 26, ähnlich Jans sen, Autobiografie, I, 70 (Bb 10; 11).

${ }^{80}$ 220-23, s. Źába, Les maximes de Ptahhotep, 45; vgl. Brunner, Weisheit, 117; Vernus, Sagesses, 86. 26.
}

lich $^{82}$. Die Biographien erstrecken sich sogar über mehr als 2500 Jahre, sie sind spätestens in der 5. Dynastie schon gut ausgebildet. Und sie leben auch länger, der letzte bis jetzt bekannte biographische Text stammt aus der Zeit Hadrians $^{83}$. Form und Thematik beider Textarten haben sich in einer so langen Zeit natürlich entsprechend den sprachlichen und gesellschaftlichen Veränderungen gewandelt. So steht im Alten Reich der König als alleiniger Verursacher allen Heils ganz im Mittelpunkt der (Laufbahn)Biographie, in den Lehren und vielen Biographien des Mittleren Reiches wird die notwendige Loyalität zum König besonders betont, in denen der Ramessidenzeit und der Dritten Zwischenzeit zeichnet sich deutlich die „Persönliche Frömmigkeit" ab.

Insgesamt aber haben Lehre und Biographie eine recht unterschiedliche Entwicklung durchgemacht. In gewisser Weise könnte man sagen, $\mathrm{da} ß$ die ägyptische Biographie sich parallel zum ägyptischen Totenkult entfaltete: Solange es schriftliche Zeugnisse (in ägyptischer Sprache) für diesen Kult gibt, solange gibt es auch Biographien. Und besonders nachhaltige Änderungen im Totenkult wirken sich auch auf die Biographien aus. So verschwinden etwa ab der Ramessidenzeit „diesseitige“ Themen (Gastmahl, Berufliches etc.) fast vollständig aus der Grabdekoration, und entsprechend werden auch die Darstellungen „weltlicher" Laufbahnen ab dieser Zeit sehr viel seltener. Der zu Beginn der 25. Dynastie (bzw. kurz vorher) einsetzende "Archaismus“ bewirkt erneut einen tiefgreifenden Wandel, der nahezu alle architektonischen, bildlichen und textlichen Erscheinungen (u. a.) des Totenkults erfaßt. Dieser Archaismus zeich-

\footnotetext{
${ }^{82}$ Zum Ansatz des Ptahhotep in die Erste Zwischenzeit vgl. jetzt Vernus, Sagesses, 21; 70-71; eine Datierung ins frühe Mittlere Reich befürwortet E. Eichler, ZÄS 128, 2001, 97-107. Auszuschließen ist es natürlich nicht, daß es schon Lehren des Alten Reiches gegeben hat, die nur der Zufall der Überlieferung nicht erhalten hat. Wenn sie keine "Klassiker" geworden wären wie viele der Lehren des Mittleren Reiches, hätten entsprechende Manuskripte tatsächlich kaum eine Chance gehabt, das Altertum zu überdauern.

${ }^{83}$ Stele Berlin 22489, s. A. Scharff, ZÄS 62, 1927 , 86-107.
} 
net sich auch in den biographischen Inschriften deutlich $\mathrm{ab}$, in denen nun der Rückgriff auf alte Formeln und Ausdrucksweisen sehr viel häufiger ist als in den vorhergehenden Epochen ${ }^{84}$.

Dagegen ist die Lehre eher mit der Geschichte der ägyptischen Verwaltung und deren Beamtentum verbunden. Das ergibt sich schon aus dem Charakter der Texte selbst, aber man hat auch andere Hinweise dafür. Bis zum Ende des Neuen Reiches ist das Bemühen um schnellen Aufstieg in der Beamtenhierarchie in den Lehren spürbar $^{85}$, während in der Lehre des Amenemope (die aus der Dritten Zwischenzeit stammen dürfte ${ }^{86}$ ) eher die Selbstbescheidung gefordert wird $^{87}$. Das könnte nicht nur durch die ,persönliche Frömmigkeit" bedingt sein, sondern auch mit der viel begrenzteren Position des ägyptischen Schreibers unter der Herrschaft der libyschen Militäraristokratie zusammenhängen. Vielleicht hat Amenemope nicht zuletzt versucht, aus dieser Not eine Tugend zu machen. In den demotischen Lehren schließlich ist vom Umgang mit hohen Staatsbeamten überhaupt keine Rede mehr. Sie sind auf den Kreis der Familie und des dörflichen Lebens begrenzt, und auch dies entspricht der Entwicklung der ägyptischen Verwaltung, die in der Perser- und Ptolemäerzeit überwiegend auf die unteren (v. a. dörflichen) Verwaltungsebenen beschränkt war.

Es ist oben (\$4) schon erwähnt worden, daß sich Lehre und Biographie ab der 19. Dynastie sprachlich auseinanderentwickeln: Die Lehren gehören zu den Texten der Alltagswelt und sind in einer Sprachstufe überliefert, die grundsätzlich der gesprochenen Sprache entspricht oder ihr doch nahesteht, die Biographien dagegen folgen den funerären Inschriften, die immer in der alten Sprachstufe (Alt- und Mittelägyptisch bzw. Spätmittelägyptisch) gehalten sind. Dies zeigt deutlich, daß die Ägypter selbst diese beiden Textarten ganz verschiedenen Ebenen zugewiesen haben. Die zunehmende Herauslösung aus der Welt des Diesseits betrifft aber nicht nur die Sprachstufe, sondern in gewisser Weise auch

\footnotetext{
${ }^{84}$ Vgl. auch Gnirs, Autobiographie, 239.

${ }^{85}$ Brunner, Weisheit, 236.

${ }^{86}$ Vgl. auch Quack, Ani, 211.

${ }^{87}$ Brunner, Weisheit, 236.
}

die Verfasser und die Adressaten der Biographien. Tempel und Gräber werden im Verlauf des Neuen Reiches, vor allem aber danach, zusehends die Domäne eines sich immer mehr spezialisierenden Berufspriestertums, die alte Einheit von Staatsbeamtem und Priester löst sich auf. Und dadurch werden Biographie und Lehre schließlich auch die Sache unterschiedlicher Berufsgruppen ${ }^{88}$. Bei dieser Lage der Dinge dürfte eine gegenseitige Beeinflussung der beiden Textarten ab der Ramessidenzeit kaum noch möglich gewesen $\operatorname{sein}^{89}$.

8. Es ist oft angenommen worden, daß Lehre und Biographie eng verwandt sind und sich gegenseitig beeinflußt haben ${ }^{90}$. Eine Beeinflussung der Biographien durch die Lehren läßt sich immerhin in einigen wenigen Fällen im früheren Mittleren Reich feststellen. In dieser Zeit erscheinen in den Biographien häufiger sentenziöse Formulierungen ${ }^{91}$, wie sie in den Lehren ganz üblich sind, und auf der Stele Kairo CG 20538 werden Teile der Loyalistischen Lehre ${ }^{92}$ im Anschluß an eine Biographie zitiert ${ }^{93}$ : Auf den kurzen biographischen Abschnitt (Z. 1-7) folgt ganz unvermittelt (Z. 8) hitj-r $m$ sb3yt jrt.n.f $h r$ $m s w . f$ und ein Auszug aus der Lehre. Auch ein biographischer Text wie der auf der Stele London UC 14333 ist in einigen Passagen recht deutlich von den Lehren geprägt ${ }^{94}$ Beide Er-

${ }^{88}$ Zwar haben auch in der Spätzeit Beamte, die vermutlich keine priesterliche Ausbildung hatten (z. B. Gaustrategen) noch Grab und Totenkult und können deshalb auch Subjekt einer Biographie sein. Sie dürften aber kaum in der Lage gewesen sein, sie richtig zu verstehen; ihr Verhältnis zu einem solchen Text war daher wohl ganz anders als in älterer Zeit.

${ }^{89}$ Gegen Vernus, Sagesses, 23.

${ }^{90} \mathrm{Vgl}$. die oben in $\int 1$ zitierten Beiträge.

${ }^{91}$ Vgl. Gnirs, Autobiographie, 207; Spiegelberg, ZÄS 45, 1908, 67-8; Vernus, RdE 28, 1976, 139-45; Jansen-Winkeln, Sentenzen und Maximen, Berlin 1999, 15-20.

${ }^{92}$ Gegen B. U. Schippers anderslautende These (ZÄS 125, 1998, 161-79) vgl. J. Quack, BiOr 57, 2000,536, n. 5 .

${ }^{93}$ Lange/Schäfer, Grab- und Denksteine des Mittleren Reiches, II, 148-9.

${ }^{94}$ S. H. Goedicke, JEA 48, 1962, 25-35; W. Schenkel, JEA 50, 1964, 6-12; Brunner, Weisheit, $370-73$. 
scheinungen tauchen dann noch einmal kurzfristig in der 18. Dynastie auf: sowohl sentenziöse Formulierungen innerhalb biographischer Inschriften $^{95}$ als auch eine Biographie, die deutlich Anklänge an Themen (v. a. Verhältnis des Sohnes zu Vater und Dienerschaft) und Formulierungen der Lehren enthält und sogar so bezeichnet wird ( $h 3 t j-{ }^{-} m$ sb3yt jrjt.n $N N$ dd f.f $m$ sb3yt $h r m s w . f)^{96}$. An sich ist es ja auch ein naheliegender Gedanke, seine (angeblich vorbildliche) Lebensführung den eigenen Kindern als "Lehre“ vorzuhalten, ebenso die Übernahme von Themen und Formulierungen dort, wo sie für eine Biographie passend sind ${ }^{97}$. Daß so etwas gelegentlich vorkommt, hat nichts Erstaunliches. Bemerkenswerter ist eigentlich die Tatsache, daß es nicht Schule gemacht hat: Es bleiben Einzelfälle, die aus dem Rahmen des Üblichen fallen, es handelte sich offenbar nur um eine kurzfristige Mode. Auch ihr Vorkommen gerade zu Beginn des Mittleren Reiches bzw. in der 12. Dynastie ist nicht verwunderlich, ist dies doch das klassische Zeitalter der Lebenslehren, in dem man geradezu erwarten sollte, daß die Verfasser der Biographien diese (neue?) Textart aufgriffen.

Umgekehrt ist ein Einfluß der Biographien auf die Lehren nur in den „biographischen“ Passagen der Königslehren erkennbar. Möglicherweise ist diese besondere Art der Lehre aus einer Verbindung der (neuen?) Textsorte „Lebenslehre" mit der Nomarchen- bzw. Königsbiographie der 11. Dynastie ${ }^{98}$ entstanden. Allerdings sind die königlichen Lehren in jeder Hinsicht ein Sonderfall: Sie haben einen anderen Adressaten als die üblichen Lebenslehren, sie

${ }^{95}$ Vgl. Spiegelberg, ZÄS 45, 1908, 68-70; Jansen-Winkeln, Sentenzen und Maximen, 15-20.

${ }_{96}$ Inschrift des Jmn-m-h3t in TT 97, s. Urk IV, 1408-11.

${ }^{97}$ Die sentenziösen Sprüche, wenn sie denn durch die Lehren angeregt sein sollten, sind nur formal, thematisch dagegen keineswegs von ihnen geprägt. In diesen Sentenzen geht es vor allem (und bis zur Spätzeit fast ausschließlich) um ein einziges Thema: die Notwendigkeit, der Toten zu gedenken und den Totenkult zu vollziehen.

${ }_{98}$ Z. B. Kairo CG 20512 des Antef Wahanch, s. W. Schenkel in: D. Arnold, Gräber des Alten und Mittleren Reiches in El-Tarif, AV 17, 1976, 52-6; Taf. 43; 53. sind nach Form und Inhalt verschieden, sie sind nur zweimal im Mittleren Reich belegt und haben keine Tradition gebildet; sie verschwinden oder werden ein Schulbuchklassiker (die Lehre des Amenemhet): die Entwicklung war eine Sackgasse. Die eigentlichen Lehren sind demgegenüber stetig den neuen Verhältnissen angepaßt worden. Eine Einwirkung der Biographien auf die Lehren in einem weiteren Sinne kann aus diesen Königslehren jedenfalls nicht erschlossen werden.

Eine andere Frage ist es, inwieweit die Entstehung der Lehren selbst durch die Biographien bedingt oder angeregt sein könnte. Die Biographie ist deutlich früher belegt als die Lehre, und vermutlich ist das nicht nur ein Zufall der Überlieferung, sondern es dürfte tatsächlich die ältere Textsorte sein. Aber daraus kann man noch nicht auf eine Abhängigkeit der jüngeren von der älteren Textsorte schließen oder gar auf eine Entstehung aus ihr, wie das in sehr dezidierter Weise gesagt worden ist: „Der ... zeitliche Ansatz der frühesten Lehren in das Mittlere Reich führt zwangsläufig [!] dazu, in der Autobiographie des Alten Reiches den Ursprung der ägyptischen Weisheitsliteratur zu suchen, da sie auf funktionaler, inhaltlicher und formaler Ebene den Raum bot, entsprechende Themen anzusprechen und allmählich zu entwickeln ${ }^{499}$. Ganz ähnlich sieht es auch A. Loprieno: ,,The autobiographies of late Old Kingdom officials represent the point of departure of the two textual forms which, once decontextualized from the Sitz im Leben of the commemoration of the single individual to the level of a collective cultural experience, acquire the status of literary genres. These two genres are wisdom texts, which expand the motifs of the so-called ,ideal autobiography ${ }^{6} . .$. , and narrative literature, ultimately related to the structure of ,career autobiography ${ }^{6}$ ... “" ${ }^{100}$. Dem dürfte die Idee zugrundeliegen, daß literarische Formen in einer Art „Diskurs“ entstehen (müßten). Ob und inwiefern die Laufbahnbiographie (auch) einen Einfluß auf die

Gnirs, Autobiographie, 208.

In: A. Loprieno (ed.), Ancient Egyptian Literature, PÄ 10, 1996, 404; übernommen auch von Vernus, Sagesses, 23. 
Entstehung der erzählenden Literatur gehabt haben könnte, sei dahingestellt. Auf jeden Fall wird man als Vorbild literarischer Narrativität in allererster Linie an mündliche Erzählungen zu denken haben ${ }^{101}$, nicht an die stocksteifen biographischen Monumentalinschriften ${ }^{102}$. Ebenso ist die Entstehung der Lehren aus der Idealbiographie bei näherer Betrachtung kein besonders naheliegender Gedanke. Es mag im Alten Reich und der Ersten Zwischenzeit einen ,weisheitlichen Diskurs" gegeben haben oder auch nicht, jedenfalls sind die "Weisheiten" von Biographie und Lehre zu unterschiedlich, als daß man an die Entstehung der einen aus der anderen glauben möchte. Diese beiden Textsorten sind eben nicht nur formal, sondern auch inhaltlich sehr verschieden, und dort, wo sie sich überschneiden, z. B. in den gemeinsamen Motiven der ,väterlichen Unterweisung, der Anerkennung von Autorität, der Charakterfestigkeit oder des menschlichen Umgangs “ ${ }^{\text {103 }}$, handelt es sich um denkbar allgemeine Dinge, die in der ägyptischen Kultur (und nicht nur in ihr) durchgehend verbindlich waren.

Sicher sind die Lehren nicht aus dem Nichts entstanden, aber deshalb ist man nicht gezwungen, einen „weisheitlichen Diskurs" anzunehmen, aus dem sie hervorgegangen sein könnten. Auch wenn sie erst ab der 12. Dynastie belegt sind und die Texte der Lehren selbst tatsächlich nie mündlich tradiert worden sein sollten ${ }^{104}$, eine Lehrtradition dieser Art (älterer Beamter unterrichtet Nachwuchsschreiber) ist mit Sicherheit schon sehr früh anzunehmen. Seit es Schreiber im Dienste der Staatsverwaltung gab, war

${ }^{101}$ Vgl. dazu auch St. Quirke, DE 16, 1990, 93 und J. Assmann, Ägypten. Eine Sinngeschichte, München 1996, 140-1.

${ }^{102}$ Es ist recht eigenartig, wie man in der Ägyptologie immer nur von dem positiv (d. h. schriftlich) Bezeugten ausgehen möchte.

83 Gnirs, Autobiographie, 208.

${ }^{104}$ So Brunner, Weisheit, 45; ebenso Assmann, „Weisheit, Schrift und Literatur im alten Ägypten“, in: A. Assmann (ed.), Weisheit, Archäologie der literarischen Kommunikation, III, München 1991, 492-3. neben und nach dem Elementarunterricht im Schreiben die weitere Erziehung zum Schreiber und Mitglied der Beamtenschaft notwendig ${ }^{105}$. Mündliche Belehrungen in diesem Milieu dürften sich inhaltlich kaum grundsätzlich von den späteren schriftlichen Lehren unterschieden haben; schließlich geht es um allgemeine Erfahrungstatsachen des menschlichen Zusammenlebens. Die Entstehung der späteren schriftlichen Lehren aus dieser mündlichen Tradition ist dann wohl im Rahmen der Entstehung der ägyptischen Literatur zu Beginn des Mittleren Reiches oder in der Ersten Zwischenzeit zu sehen. Ein weiterer Impuls dazu könnte das Bemühen der Könige der 12. Dynastie um eine gewisse „Standardisierung" der Erziehung gewesen sein, um eine einheitlich in ihrem Sinne ausgebildete Elite zu schaffen. Bei dieser Umsetzung einer älteren mündlichen Lehrtradition ins Schriftliche und Literarische könnten und sollten sich zwar gewisse Veränderungen ergeben haben. Einen Einfluß der Biographie in diesem Prozeß anzunehmen, erscheint mir aber keineswegs zwingend, sondern im Gegenteil recht unwahrscheinlich.

\section{SUMMARY}

Contrary to current opinion, Egyptian instructions and (auto-)biographies do not have much in common with each other. The differences between the two are much more fundamental. This includes their formal structure, use and purpose, literary character, and - beginning with the later New Kingdom - the linguistic idiom they employ. Instructions are written in Late Egyptian and Demotic, biographies retain the "classical" Middle Egyptian. Both kinds of texts also diverge with regard to the content. The range of topics which they treat overlap only partially, and even comparable events are reported from a different perspective. In view of these differences, it seems rather improbable that the biographies had an important influence on the genesis of the instructions.

${ }^{105}$ So wie ein Handwerkerlehrling bei seinem Meister seine handwerklichen Fähigkeiten und die Regeln der Zunft lernte. 